\title{
Prognostic factors of severe community-acquired staphylococcal pneumonia in France
}

\author{
Yves Gillet ${ }^{1,2,3}$, Anne Tristan ${ }^{2,3,6}$, Jean-Philippe Rasigade $\mathbb{B}^{2,3,6}$, Mitra Saadatian-Elahi ${ }^{4}$, Coralie Bouchiat ${ }^{2,3}$, \\ Michele Bes ${ }^{2,3}$, Oana Dumitrescu ${ }^{2,3}$, Marie Leloire ${ }^{2,3}$, Céline Dupieux ${ }^{2,3}$, Frédéric Laurent ${ }^{2,3}$, Gérard Lina ${ }^{2,3}$, \\ Jerome Etienne ${ }^{2,3}$, Philippe Vanhems $s^{3,4}$, Laurent Argaud $\mathbb{1}^{5}$, Francois Vandenesch $\mathbb{1}^{2,3}$ and \\ the PVL pneumonia study group ${ }^{7}$
}

\begin{abstract}
${ }^{1}$ Paediatric Emergency and Intensive Care Unit, Hospices Civils de Lyon, Lyon, France. ${ }^{2}$ French Reference Centre for Staphylococci, Institute for Infectious Agents, Hospices Civils de Lyon, Lyon, France. ${ }^{3} \mathrm{CIRI}$, Centre International de Recherche en Infectiologie, Université de Lyon, Inserm, U1111, Université Claude Bernard Lyon 1, CNRS, UMR5308, ENS de Lyon, Lyon, France. ${ }^{4}$ Infection Control and Epidemiology Dept, Hospices Civils de Lyon, Lyon, France. ${ }^{5}$ Medical Intensive Care Unit, Hospices Civils de Lyon, Lyon, France. ${ }^{6}$ These authors contributed equally to the work. ${ }^{7} \mathrm{~A}$ complete list of PVL pneumonia study group members is listed at the end of this article.
\end{abstract}

Corresponding author: Francois Vandenesch (francois.vandenesch@univ-lyon1.fr)

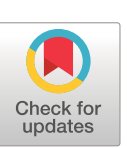

This version is distributed under the terms of the Creative Commons Attribution Non-Commercial Licence 4.0. For reproduction rights and permissions contact permissions@ersnet.org

This article has supplementary material available from erj.ersjournals.com

Received: 8 Dec 2020 Accepted: 31 March 2021

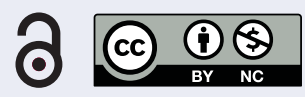

Shareable abstract (@ERSpublications)

In severe community-acquired staphylococcal pneumonia, Panton-Valentine leukocidin is associated with pleuropneumonia in toddlers and, in older patients, with increased mortality independent of methicillin resistance and other virulence factors https://bit.ly/3dQ5Dgm

Cite this article as: Gillet Y, Tristan A, Rasigade J-P, et al. Prognostic factors of severe communityacquired staphylococcal pneumonia in France. Eur Respir J 2021; 58: 2004445 [DOI: 10.1183/ 13993003.04445-2020].

\section{Abstract}

Purpose Staphylococcus aureus causes severe forms of community-acquired pneumonia (CAP), namely staphylococcal pleuropneumonia in young children and staphylococcal necrotising pneumonia in older patients. Methicillin resistance and the Panton-Valentine leukocidin (PVL) toxin, as well as less specific factors, have been associated with poor outcome in severe CAP, but their roles are unclear.

Methods A prospective multicentre cohort study of severe staphylococcal CAP was conducted in 77 paediatric and adult intensive care units in France between January 2011 and December 2016. After ageclustering, risk factors for mortality, including pre-existing conditions, clinical presentation, laboratory features, strain genetic lineage, PVL, other virulence factors and methicillin resistance were assessed using univariate and multivariable Cox and LASSO (least absolute shrinkage and selection operator) regressions. Results Out of 163 included patients, aged 1 month to 87 years, 85 (52.1\%) had PVL-positive CAP; there were 20 (12.3\%) patients aged <3 years (hereafter “toddlers”), among whom 19 (95\%) had PVL-positive CAP. The features of PVL-positive CAP in toddlers matched with the historical description of staphylococcal pleuropneumonia, with a lower mortality (three (15\%) out of 19) compared to PVL-positive CAP in older patients (31 (47\%) out of 66). Mortality in older patients was predicted by PVL-positivity (hazard ratio (HR) 1.81, 95\% CI 1.03-3.17) and methicillin resistance (HR 2.37, 95\% CI 1.29-4.34) independently from $S$. aureus lineages and the presence of other determinants of virulence.

Conclusion PVL was associated with staphylococcal pleuropneumonia in toddlers and was a risk factor for mortality in older patients with severe CAP, independently of methicillin resistance, $S$. aureus genetic background and other virulence factors.

\section{Introduction}

Staphylococcal necrotising pneumonia caused by Staphylococcus aureus was first described in 1919 in adults during the influenza pandemic [1]. It is associated with airway haemorrhage, epithelial necrosis and a high fatality rate in otherwise healthy patients [2]. It was seldom reported during the 20th century [3, 4], until the epidemiological and pathophysiological links with Panton-Valentine leukocidin (PVL) were discovered at the turn of the century $[2,5]$. Subsequent reports confirmed the high fatality rates $(40-50 \%)$ of PVL-associated pneumonia in adults [6-9]. 
Independent of staphylococcal necrotising pneumonia, staphylococcal pleuropneumonia in young children was described in the late 1950s and 1960s as a specific clinical entity characterised by round-shaped lung infiltrations evolving towards bullous lesions, purulent pleural effusion and a lack of airway haemorrhage or epithelial necrosis [10, 11]. Recent case series of pleuropneumonia involving PVL-positive community-acquired $S$. aureus $[12,13]$ pointed to a possible relationship between pleuropneumonia and PVL; mortality was $<5 \%$ in these series.

The striking differences between pleuropneumonia and necrotising pneumonia, both associated with PVL, but exhibiting contrasting mortality rates, may have contributed to controversies regarding the impact of PVL on the severity of staphylococcal pneumonia [14-16]. In this context, whether age affects both the clinical picture and the outcome of community-acquired PVL-positive staphylococcal pneumonia, which factors are associated with severity and whether PVL is an independent factor of severity are questions that remain to be answered and which are addressed herein.

Patients and methods

\section{Ethics}

The regional ethics committee approved the study (number: A11-39). Written informed consent was obtained from all patients or their parents.

\section{Study design and participants}

This is a prospective, multicentre, observational cohort of patients with $S$. aureus community-acquired pneumonia (CAP) requiring intensive care. All French adult and paediatric intensive care units (ICU) were contacted via the main learned medical societies (Société de Réanimation de Langue Française: 200 adult ICU; Groupe Francophone de Réanimation et d’Urgence Pédiatrique of the Société Française de Pédiatrie: 41 paediatric ICU) and invited to participate. Patients with $S$. aureus CAP (see criteria in supplementary methods) and admitted to a participating ICU were included between January 2011 and December 2016. Exclusion criteria were HIV-positive status, hospital-acquired pneumonia or admission to hospital in the past 3 months. Clinical, laboratory and therapeutic data were collected prospectively at admission to ICU and on day 1, 3 and 7. Severity was evaluated using age-adapted severity scores (see details in the supplementary methods).

\section{Microbiology}

The causative staphylococcal isolates were transmitted to the French National Reference Centre for Staphylococci for full characterisation and DNA array genotyping (supplementary methods) [17].

\section{Statistical analysis}

We determined whether the previous descriptions of different PVL-associated diseases, namely pleuropneumonia in children and staphylococcal necrotising pneumonia in adults correlated with identifiable age groups in patients with severe CAP. To this aim, patterns in patient age distribution were detected using a clustering procedure (supplementary figure S1). Following this, disease presentation and outcome in toddlers (age $<3$ years, as determined by the age clustering procedure) were compared to that found in older patients. The comparison of PVL-positive and PVL-negative cases was restricted to older patients (aged $\geqslant 3$ years) because $95 \%$ of toddlers had PVL-positive CAP. Between-group comparisons used Fisher's exact test or t-test, as appropriate. Possibly nonlinear changes of mortality rates in function of patient age were visualised using kernel density estimation with bootstrap confidence intervals (supplementary methods). Potential predictors of mortality were investigated using univariate and multivariable Cox proportional hazards models. To avoid bias due to row-wise deletion of cases containing missing data in multivariate models [18], all missing data were imputed prior to analysis using a nonlinear, random forest-based multiple imputation technique. Imputation uncertainty was accounted for by replacing binary factors (such as leukopenia, for example) by a probability estimate between 0 (absence) and 1 (presence).

To further examine the relationship between microbiological characteristics and outcome, we assessed whether the isolate's lineage and the presence of virulence factors, as determined using DNA arrays, predicted mortality and major clinical characteristics at admission, namely, rash, haemoptysis and leukopenia. These analyses were restricted to adult patients. All models included the Charlson score as a covariate to account for the diversity of age and baseline health in the cohort. Models with death as the response variable used Cox regression, with coefficients reported as hazard ratios. Models with rash, haemoptysis or leukopenia as the response variable used logistic regression, with coefficients reported as odds ratios. In a first series of models, each outcome was regressed on the clonal complex, treated as a categorical variable, to identify associations of the bacterial lineage with clinical presentation. Given the 
strong genetic diversity in our collection, lineages with <10 isolates were pooled into an "other" category before regression analysis. In a second series of models, we examined a near-exhaustive array of virulence determinants for associations with death, rash, haemoptysis and leukopenia. A subset of the 332 loci and alleles, as determined using DNA arrays, was selected a priori based on their plausible role in virulence (e.g. excluding antimicrobial resistance determinants other than mecA) and nonredundancy (e.g. excluding the lukF component of $p v l$ which is virtually always present with the lukS component; supplementary table S8). The alleles defining agr groups I to IV were also included, but not the lineage (clonal complex) which would be redundant with the genotypic profile. Variables present or absent in $<10$ isolates were excluded. A final set of 28 nonconstant genotypic determinants were included in the final analyses (supplementary table S8). Determinants were coded as 0 when absent and 1 when present. Ambiguous DNA array results (missing data, $0.8 \%$ of results) were imputed using the random forest procedure described earlier. Given the large number of determinants, we used sparse regression (least absolute shrinkage and selection operator (LASSO)) as implemented in the glmnet R package, to identify the determinants retained in sparse models [19]. Briefly, LASSO models are built sequentially by modifying a L1 penalisation constraint such that more penalised models include fewer predictors. The best-fitting models (using death, rash, haemoptysis or leukopenia as the response variable) were selected using leave-one-out cross-validation.

Analyses were conducted using R software v3.6.0 (www.R-project.org/). Anonymised data and software code required to reproduce the results are available at github.com/rasigadelab/severecap.

Results

Clustering analysis of patient age identifies specific patterns in PVL-positive and PVL-negative S. aureus pneumonia

A total of 228 patients were eligible. After removal of those who did not comply with the inclusion criteria, those who did not provide consent and those with missing case report forms, 163 patients were included (supplementary figure S1). The included patients were hospitalised in 77 ICUs and aged from 1 month to 87 years. 85 patients (52.1\%) were infected by a PVL-positive S. aureus. Clustering by age of patients with PVL-positive CAP identified two nonoverlapping clusters aged $<3$ years and $\geqslant 3$ years (supplementary figure S2). In patients with PVL-negative CAP, we identified two overlapping clusters centred at ages 28 years and 60 years. Almost all cases in patients aged $<3$ years (hereafter "toddlers") were PVL-positive, while PVL positivity in older patients was evenly distributed according to age (supplementary figure S2). In contrast, PVL-negative CAP was exceptional in younger patients (only one case aged $<15$ years), and its prevalence increased with patient age, peaking at 60 years. Hence, toddlers accounted for $12.3 \%$ of all severe staphylococcal CAP in our cohort, and $22.3 \%$ of PVL-positive cases. The microbiological and clinical features of CAP differed markedly between toddlers and older patients (supplementary table S1). PVL positivity was more frequent in toddlers, more strongly so than methicillin resistance (seven out of 20 versus 23 out of 143; OR 2.8, 95\% CI 0.85-8.6). Additional details on the specificities observed in toddlers are given in the supplementary results.

\section{Mortality according to age}

Mortality increased with age, reaching a plateau at 30 years in PVL-positive cases (figure 1). Age-dependent increase of mortality was more linear in PVL-negative cases (supplementary figure S3). Mortality was lower in toddlers than older patients (three out of 20 versus 52 out of 143; OR 0.31, 95\% CI 0.06-1.15). In toddlers with PVL-positive CAP (supplementary figure S3c and d), most cases occurred before 12 months of age and were nonlethal, while mortality increased after 1 year of age.

Since only one toddler had PVL-negative CAP, we could not compare the outcomes of PVL-positive and -negative CAP in this group. Further comparisons according to PVL positivity and analysis of severity factors were restricted to patients aged $\geqslant 3$ years to avoid the interpretation bias that would have resulted from the pooling of such markedly different patients (namely, toddlers and older patients) in a single analysis.

PVL is associated with specific symptoms and is a severity factor in patients aged $\geqslant 3$ years Among the 143 patients aged $\geqslant 3$ years, 66 (46.2\%) had PVL-positive CAP. Compared to PVL-negative CAP, patients with PVL-positive CAP were younger and less likely to have an underlying condition (table 1). At admission, they had more frequent airway haemorrhage and cutaneous eruption or rash evoking immune system reaction. In addition, PVL-positive CAP patients were more likely to present with septic shock, leukopenia, elevated blood lactate and Sequential Organ Failure Assessment (SOFA) severity score, and to require extracorporeal membrane oxygenation (ECMO). 

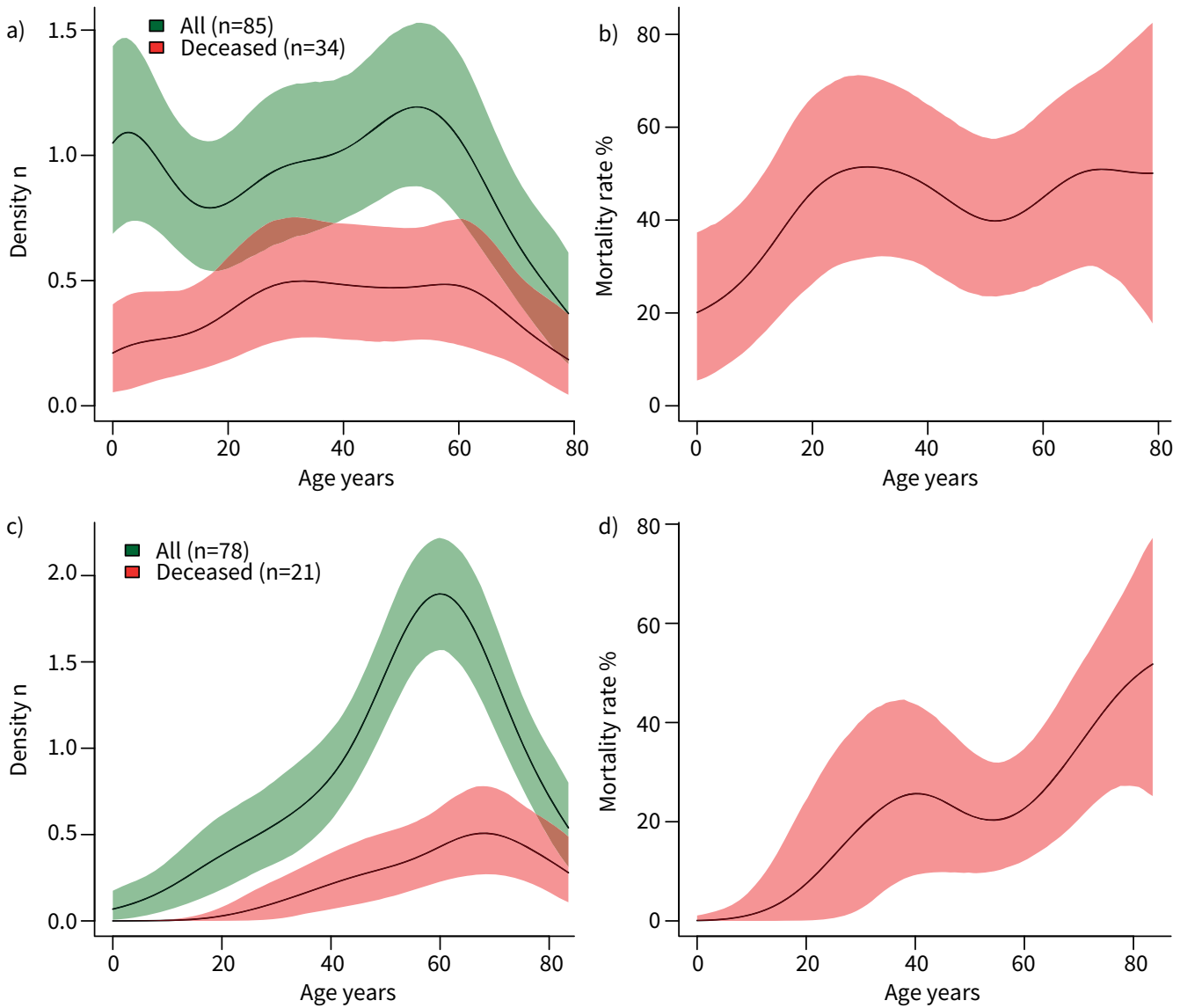

FIGURE 1 Mortality of patients with Staphylococcus aureus community-acquired pneumonia (CAP) according to age and the presence of the Panton-Valentine leukocidin (PVL). a, c) Density estimates of the occurrence of pneumonia in all patients of the considered group and in deceased patients. b, d) Estimates of mortality rate. Shaded areas present bootstrap-based 95\% confidence bands of the estimates. Analyses were conducted separately for patients with a, b) PVL-positive CAP and c, d) PVL-negative CAP.

Appropriate initial antibiotic treatment, defined as one or more drugs active against the corresponding strain, was equally likely in PVL-positive and -negative CAP. However, the initial treatment was less frequently appropriate in methicillin-resistant S. aureus (MRSA) cases (OR 0.027, 95\% CI 0.006-0.097). See the supplementary results for additional information on antibiotic treatment.

Most of the differences observed between patients with PVL-positive and -negative CAP at admission persisted during the first week in ICU (tables 2 and 3). The higher SOFA score at admission persisted at day 1 , as did the higher median blood lactate. Temperature $>39^{\circ} \mathrm{C}$, airway haemorrhage and rash remained significantly more common in PVL-positive cases, who received more frequently inhaled nitric oxide and required ECMO support.

\section{Survival}

We used survival analysis to examine the influence of microbiological and clinical factors on lethal outcome in the 143 patients aged $\geqslant 3$ years. Kaplan-Meier survival curves suggested that PVL-positivity and methicillin resistance contributed additively to mortality (figure 2); median (interquartile range (IQR)) survival time was 1 day (0-7 days) for PVL-positive cases and 7 days (3-14 days) for PVL-negative cases ( $p=0.02$, Mann-Whitney U-test). Among the 91 survivors, length of ICU stay was longer in those with PVL-positive CAP (median, IQR time until discharge 39 days, 26-68 days) than in those with PVL-negative CAP (29 days, 20-42 days; p=0.01, Mann-Whitney U-test).

We then used bivariate and multivariable Cox proportional hazards models to identify potential independent risk factors for mortality (table 4). Predictors were included in the models based on their 
TABLE 1 Clinical and laboratory characteristics at admission according to Panton-Valentine leukocidin (PVL) status in patients aged $\geqslant 3$ years

\begin{tabular}{|c|c|c|c|c|c|}
\hline & \multicolumn{2}{|c|}{ PVL-positive S. aureus } & \multicolumn{2}{|c|}{ PVL-negative $S$. aureus } & \multirow[t]{2}{*}{ p-value } \\
\hline & Patients & Missing & Patients & Missing & \\
\hline Patients & 66 & & 77 & & \\
\hline $\begin{array}{l}\text { Delay in days between onset and } \\
\text { ICU admission }\end{array}$ & $3.39 \pm 3.74$ & 0 & $4.64 \pm 4.80$ & 4 & 0.092 \\
\hline Female/male & $31 / 35$ & 0 & $27 / 50$ & 0 & 0.173 \\
\hline Age years & $47(28.7-60.2)$ & 0 & $58(48-67)$ & 0 & $<0.001$ \\
\hline Children age $<18$ years & $6(9.1)$ & 0 & $1(1)$ & 0 & 0.049 \\
\hline MRSA & $14(21.2)$ & 0 & $9(11.7)$ & 0 & 0.170 \\
\hline Absence of underlying condition & $39(59.1)$ & 0 & $22(28.6)$ & 0 & $<0.001$ \\
\hline Charlson's score & $1.18 \pm 0.18$ & 0 & $2.14 \pm 0.22$ & 0 & 0.001 \\
\hline Temperature $>39^{\circ} \mathrm{C}$ & $35(53)$ & 0 & $29(38.2)$ & 1 & 0.092 \\
\hline Airway haemorrhage & $29(43.9)$ & 0 & $18(23.4)$ & 0 & 0.012 \\
\hline Toxin-mediated rash & $14(21.2)$ & 0 & $5(6.6)$ & 1 & 0.013 \\
\hline Bilateral pneumonia & $46(70.8)$ & 1 & $52(68.4)$ & 1 & 0.855 \\
\hline Significant pleural effusion` & $3(4.5)$ & 0 & $1(1.4)$ & 3 & 0.343 \\
\hline Leukocytes $\mathrm{G} \cdot \mathrm{L}^{-1}$ & $4.60(1.4-15)$ & 1 & $9.34(5-15.8)$ & 3 & 0.011 \\
\hline Leukopenia $<3 \mathrm{G} \cdot \mathrm{L}^{-1}$ & $28(43.1)$ & 1 & $14(18.9)$ & 3 & 0.003 \\
\hline Blood lactate $\mathrm{mg} \cdot \mathrm{L}^{-1}$ & $3.95(2.3-5.5)$ & 8 & $2.4(1.7-4.6)$ & 9 & 0.015 \\
\hline CRP $\mathrm{mg} \cdot \mathrm{L}^{-1}$ & $327.5(191.2-396.7)$ & $26^{+}$ & $251.6(127-351)$ & $18^{+}$ & 0.041 \\
\hline Procalcitonin $\mu \mathrm{g} \cdot \mathrm{L}^{-1}$ & $137.5(51.7-488.5)$ & $40^{+}$ & $57(8-200)$ & $58^{+}$ & 0.032 \\
\hline Appropriate antibiotics & $47(75.8)$ & 4 & $62(80.5)$ & 0 & 0.539 \\
\hline
\end{tabular}

Data are presented as $n$, mean \pm sD, median (interquartile range) or $n(\%)$, unless otherwise stated. Percentages are calculated among those with data. S. aureus: Staphylococcus aureus; ICU: intensive care unit; MRSA: methicillin-resistant S. aureus; CRP: C-reactive protein. \#: underlying conditions include malignancies, tobacco smoking, alcohol abuse, diabetes and body mass index $>30 \mathrm{~kg} \cdot \mathrm{m}^{-2}$; ${ }^{\bullet}$ : needing drainage; ${ }^{+}$: inflammatory markers (CRP or procalcitonin) are missing for 14 patients (seven in each group).

expected relevance to disease outcome. In addition to PVL and methicillin resistance, these included baseline patient characteristics, namely sex and the Charlson comorbidity score; characteristics reflecting 1) severity upon admission, namely the SOFA score, haemoptysis, leukopenia and blood lactates, 2) inflammatory reaction, namely rash and blood procalcitonin and 3) a risk factor for staphylococcal superinfection of the lung, namely a flu-like illness; and the appropriateness and expected toxin-suppressing activity of the antibiotics received (see the supplementary results for details). In the best-fitting multivariable model, the independent predictors of death were methicillin resistance, haemoptysis, rash, leukopenia, elevated blood lactates and the absence of a flu-like illness (supplementary table S3).

TABLE 2 Clinical and laboratory characteristics during stay according to Panton-Valentine leukocidin (PVL) status in patients aged $\geqslant 3$ years

\begin{tabular}{|c|c|c|c|c|c|}
\hline & \multicolumn{2}{|c|}{ PVL-positive S. aureus } & \multicolumn{2}{|c|}{ PVL-negative $S$. aureus } & \multirow[t]{2}{*}{$\mathrm{p}$-value } \\
\hline & Patients & Missing & Patients & Missing & \\
\hline Patients & 66 & & 77 & & \\
\hline Temperature $>39^{\circ} \mathrm{C}$ & $44(75.9)$ & 8 & $44(58.7)$ & 2 & 0.043 \\
\hline Airway haemorrhage & $35(59.3)$ & 7 & $28(37.3)$ & 2 & 0.015 \\
\hline Toxin-mediated rash & $15(25.4)$ & 7 & $8(10.7)$ & 2 & 0.037 \\
\hline Bilateral pneumonia & $45(78.9)$ & 9 & $61(83.6)$ & 4 & 0.505 \\
\hline Significant pleural effusion ${ }^{\#}$ & $10(16.4)$ & 5 & $10(13.7)$ & 4 & 0.808 \\
\hline Highest CRP $\mathrm{mg} \cdot \mathrm{L}^{-1}$ & $352(230-447)$ & 38 & $228(106.0-347)$ & 38 & 0.017 \\
\hline Highest procalcitonin $\mu \mathrm{g} \cdot \mathrm{L}^{-1}$ & $91(28.5-375)$ & 37 & $35.4(3.3-98.6)$ & 47 & 0.032 \\
\hline
\end{tabular}


TABLE 3 Severity scores and markers at admission and during evolution according to Panton-Valentine leukocidin (PVL) status in patients aged $\geqslant 3$ years

\begin{tabular}{|c|c|c|c|c|c|}
\hline & \multicolumn{2}{|c|}{ PVL-positive S. aureus } & \multicolumn{2}{|c|}{ PVL-negative $S$. aureus } & \multirow[t]{2}{*}{$\mathrm{p}$-value } \\
\hline & Patients & Missing & Patients & Missing & \\
\hline Patients & 66 & & 77 & & \\
\hline \multicolumn{6}{|l|}{ Admission } \\
\hline SOFA score & $8.9 \pm 5.68$ & 2 & $6.9 \pm 5.01$ & 8 & 0.004 \\
\hline Septic shock & $31(48.4)$ & 4 & $22(30.1)$ & 2 & 0.035 \\
\hline ARDS & $20(32.8)$ & 7 & $16(22.9)$ & 5 & 0.352 \\
\hline Severe ARDS & $12(19.7)$ & 7 & $10(14.3)$ & 5 & 0.485 \\
\hline Invasive ventilation & $32(48.5)$ & 0 & $31(42.5)$ & 4 & 0.499 \\
\hline ECMO & $11(16.9)$ & 1 & $3(4.2)$ & 5 & 0.022 \\
\hline \multicolumn{6}{|l|}{ Day 1} \\
\hline SOFA score & $10.9 \pm 5.96$ & 14 & $8.8 \pm 5.25$ & 8 & 0.040 \\
\hline Septic shock & $31(54.4)$ & 9 & $28(40.6)$ & 8 & 0.152 \\
\hline ARDS & $27(50)$ & 12 & $32(50)$ & 13 & 1 \\
\hline Severe ARDS & $21(38.9)$ & 12 & $19(29.7)$ & 13 & 0.332 \\
\hline Death & $7(10.6)$ & 0 & $4(5.2)$ & 0 & $<0.001$ \\
\hline Lactate variation ${ }^{\#}$ & $0.62 \pm 4.38$ & 19 & $-0.69 \pm 2.10$ & 14 & 0.063 \\
\hline \multicolumn{6}{|l|}{ Day 3} \\
\hline SOFA score & $10.6 \pm 6.51$ & 24 & $8.1 \pm 5.34$ & 8 & 0.045 \\
\hline Cumulative death & $17(25.8)$ & 0 & $7(9.1)$ & 0 & $<0.001$ \\
\hline \multicolumn{6}{|l|}{ Day 7} \\
\hline SOFA score & $8 \pm 6.84$ & 29 & $6.8 \pm 5.70$ & 16 & 0.347 \\
\hline Cumulative death & $23(34.8)$ & 0 & $10(13)$ & 0 & $<0.001$ \\
\hline \multicolumn{6}{|l|}{ During whole ICU stay } \\
\hline Invasive ventilation & $56(84.8)$ & 0 & $60(81.1)$ & 3 & 0.655 \\
\hline Inhaled NO & $21(33.3)$ & 3 & $11(15.3)$ & 5 & 0.016 \\
\hline ECMO & $20(32.3)$ & 4 & $8(11)$ & 4 & 0.003 \\
\hline Death & $31(47.0)$ & 0 & $21(27.3)$ & 0 & 0.023 \\
\hline
\end{tabular}

Data are presented as $\mathrm{n}, \mathrm{n}(\%)$ or median (interquartile range), unless otherwise stated. Percentages are calculated among those with data. S. aureus: Staphylococcus aureus; SOFA: Sequential Organ Failure Assessment; ARDS: acute respiratory distress syndrome; ECMO: extracorporeal membrane oxygenation; ICU: intensive care unit. \#: between day 0 and day 1 ; ${ }^{\uparrow}$ : from day 0 to day 3 or day 7. 
TABLE 4 Cox regression analysis of predictors of death in patients aged $\geqslant 3$ years with Staphylococcus aureus pneumonia, including clinical predictors at admission and microbiological predictors

\begin{tabular}{|c|c|c|c|}
\hline & Bivariate models & Full model & Best-fitting model \\
\hline Charlson comorbidity score (per point) & $1.05(0.91-1.21)$ & $1.03(0.85-1.26)$ & \\
\hline Male sex & $0.68(0.39-1.17)$ & $0.95(0.51-1.78)$ & \\
\hline PVL & $1.95(1.12-3.41)$ & $0.87(0.42-1.83)$ & \\
\hline Methicillin resistance & $2.57(1.41-4.69)$ & $3.86(1.34-11.16)$ & $2.87(1.53-5.40)$ \\
\hline SOFA score & $1.11(1.05-1.16)$ & $1.03(0.96-1.11)$ & \\
\hline Flu-like illness & $0.71(0.41-1.23)$ & $0.32(0.15-0.68)$ & $0.32(0.17-0.60)$ \\
\hline Haemoptysis & $2.62(1.52-4.52)$ & $1.87(0.98-3.54)$ & $2.15(1.17-3.93)$ \\
\hline Rash & $2.17(1.12-4.23)$ & $2.59(1.22-5.48)$ & $2.52(1.24-5.12)$ \\
\hline Leukopenia <3 G. $\mathrm{L}^{-1}$ & $3.45(2.00-5.97)$ & $2.38(1.19-4.76)$ & $2.38(1.23-4.58)$ \\
\hline Procalcitonin per two-fold increase & $1.40(1.21-1.63)$ & $1.05(0.88-1.24)$ & \\
\hline Lactates per two-fold increase & $2.40(1.85-3.12)$ & $2.23(1.52-3.28)$ & $2.64(1.95-3.57)$ \\
\hline Adapted antimicrobial therapy & $0.82(0.44-1.53)$ & $1.54(0.52-4.59)$ & \\
\hline Antitoxin therapy & $2.67(1.48-4.81)$ & $1.29(0.63-2.65)$ & \\
\hline
\end{tabular}

Data are presented as hazard ratio $(95 \% \mathrm{Cl})$. Bivariate models were independent Cox proportional hazards models, one per predictor. The full multivariable model included all predictors (likelihood ratio test, $p<0.001$; Akaike information criterion (AIC) 420 with 13 degrees of freedom). The best-fitting model was obtained using a stepwise procedure, starting from the full model, and minimising the AIC (409 with 6 degrees of freedom). Confidence interval widths were not corrected for test multiplicity. PVL: Panton-Valentine leucocidin; SOFA: Sequential Organ Failure Assessment.

Interestingly, PVL positivity significantly predicted death in bivariate analysis (table 4) while the best-fitting multivariable model included previously recognised severity factors in PVL-positive lung infection, namely haemoptysis and leukopenia [20], but not PVL itself. The exclusion of PVL, but not its clinical consequences from the final model is suggestive of a chain of consequence between PVL, severity factors and death, where the severity factors mediate the effect of PVL on death [21]. To verify this interpretation, we constructed an additional multivariable Cox regression model including only microbiological and baseline predictors, but not the potential consequences of PVL such as treatment or severity factors. In this model, PVL positivity predicted death (adjusted hazard ratio (HR) 1.81, 95\% CI 1.04-3.17) independent of methicillin resistance, sex, Charlson score and flu-like illness. Thus, PVL positivity was a risk factor of mortality independent of other baseline factors. See the supplementary results and supplementary figure S4 for additional data on severity-associated features and on interactions between predictors of mortality.

\section{Contribution of the strain genetic background and other virulence factors to severity}

Although PVL and methicillin resistance have been repeatedly associated with poor outcome in previous studies, it is still debated whether they exert a direct effect on outcome or rather act as surrogate markers for more virulent lineages. To examine this issue, we tested whether mortality and major severity factors at admission, namely, rash, haemoptysis and leukopenia, could be predicted by the isolate's lineage (supplementary table S2) and virulence gene content, independently of the Charlson comorbidity score.

In models using lineage as a predictor (table 5), the Charlson score predicted leukopenia, but not death, rash or haemoptysis, while the isolate's lineage contributed to predict rash, possibly leukopenia $(\mathrm{p}=0.06$, analysis of deviance Chi-squared test), but not death or haemoptysis. Substantial associations between lineage and outcome were found, namely between CC121 and a rash, and between CC152, CC398 and leukopenia (table 5). In an additional, mixed-effect Cox regression including one random intercept per lineage, both mecA (HR 2.25, 95\% CI 1.18-4.27) and pvl (HR 1.98, 95\% CI 1.08-3.62) were independent predictors of death. Collectively, these results indicate that the $S$. aureus lineage per se does not predict death independently of mecA and $p v l$, which favours a direct role of these determinants rather than an indirect role as lineage markers. However, other clinical characteristics such as rash or leukopenia were influenced by the strain's lineage, suggesting a potential role of other virulence factors.

To further decipher the relationships between virulence and CAP outcome, we examined 28 virulence determinants for associations with death, rash, haemoptysis and leukopenia (see supplementary figure S5 for correlation between the selected predictors). The best-fitting LASSO regression models for each outcome, selected using leave-one-out cross-validation, are reported in table 6 (see supplementary figure 
TABLE 5 Association of Staphylococcus aureus lineage with death, rash, haemoptysis and leukopenia in 143 adult patients with severe community-acquired pneumonia

\begin{tabular}{lcccc} 
& \multicolumn{4}{c}{ Regression coefficient } \\
\cline { 2 - 5 } & $\begin{array}{c}\text { Death } \\
\text { HR }(95 \% \mathrm{CI})\end{array}$ & $\begin{array}{c}\text { Rash } \\
\text { OR }(95 \% \mathrm{CI})\end{array}$ & $\begin{array}{c}\text { Haemoptysis } \\
\text { OR }(95 \% \mathrm{CI})\end{array}$ & $\begin{array}{c}\text { Leukopenia } \\
\text { OR }(95 \% \mathrm{CI})\end{array}$ \\
\hline Charlson comorbidity score & $1.11(0.95-1.29)$ & $0.90(0.61-1.32)$ & $0.97(0.78-1.21)$ & $0.80(0.62-1.04)$ \\
CC121 & $1.71(0.66-4.42)$ & $24.19(4.85-120.48)$ & $0.68(0.16-2.90)$ & $0.90(0.16-4.97)$ \\
CC152 & $2.55(1.07-6.09)$ & $0.47(0.05-4.61)$ & $3.30(0.96-11.29)$ & $7.84(2.06-29.81)$ \\
CC30 & $0.69(0.25-1.89)$ & $0.87(0.15-4.95)$ & $0.68(0.21-2.23)$ & $1.17(0.31-4.40)$ \\
CC398 & $1.07(0.39-2.94)$ & $0.62(0.07-5.62)$ & $1.04(0.30-3.58)$ & $4.47(1.22-16.34)$ \\
CC5 & $0.38(0.09-1.66)$ & व & $0.56(0.14-2.33)$ & $0.79(0.15-4.31)$ \\
CC8 & $1.03(0.34-3.11)$ & - & $0.53(0.10-2.88)$ & $2.62(0.52-13.15)$ \\
CC80 & $1.59(0.58-4.35)$ & $0.94(0.10-9.13)$ & $1.39(0.34-5.68)$ & $3.47(0.77-15.58)$ \\
Charlson comorbidity score ${ }^{\#}$ & $\mathrm{p}=0.54$ & $\mathrm{p}=0.45$ & $\mathrm{p}=0.32$ & $\mathrm{p}=0.02$ \\
Lineage $^{\#}$ (CC) & $\mathrm{p}=0.15$ & $\mathrm{p}<0.001$ & $\mathrm{p}=0.35$ & $\mathrm{p}=0.01$ \\
\hline
\end{tabular}

Lineages with sample size $<10$ were pooled and used as the reference category. Death prediction model used multivariable Cox regression, with coefficients reported as hazard ratios (HRs). Other models used multivariable logistic regression, with coefficients reported as odds ratios. ": analysis of deviance, Chi-squared test; ฯ: unresolved coefficient due to small sample size.

S5 and supplementary tables S4 to S7 for details of LASSO models). This analysis confirmed that both mecA and pvl were independent predictors of death, even when all other virulence markers were considered. In addition, the pvl was an independent predictor of haemoptysis and leukopenia, but not rash. Rash was mainly predicted by an agrIV background, in line with the association of rash with lineage CC121 of the agrIV background (table 5). Of note, PVL remained among the top-ranking predictors not included in the best-fitting model (supplementary table S5).

The LASSO models of haemoptysis and leukopenia yielded additional insights into the interplay of virulence factors with CAP presentation. In addition to PVL, edinB (encoding the epidermal differentiation

TABLE 6 Association of Staphylococcus aureus virulence determinants with death, rash, haemoptysis and leukopenia in 143 adult patients with severe community acquired pneumonia

\begin{tabular}{|c|c|c|c|c|}
\hline & \multicolumn{4}{|c|}{ Coefficient in best-fitting LASSO model } \\
\hline & Death HR & Rash OR & Haemoptysis OR & Leukopenia OR \\
\hline Charlson score (per point) & - & - & - & 0.87 \\
\hline mecA & 1.89 & - & - & - \\
\hline agrl & - & - & - & 1.52 \\
\hline agrlII & - & - & - & 0.79 \\
\hline agrlV & - & 13.99 & - & - \\
\hline lukS-PV & 1.23 & - & 1.15 & 2.52 \\
\hline edin B & - & - & 2.27 & 1.60 \\
\hline cap5 & - & 0.63 & - & 1.78 \\
\hline etD & - & - & - & 2.32 \\
\hline sea & - & - & - & 2.05 \\
\hline$e g c$ & - & - & - & 0.92 \\
\hline lukD & - & - & - & 0.53 \\
\hline icaC & 0.81 & - & - & - \\
\hline cna & - & 1.31 & - & - \\
\hline eap & 0.86 & - & - & - \\
\hline$s d r D$ & 0.99 & - & - & - \\
\hline \multicolumn{5}{|c|}{$\begin{array}{l}\text { Models with least absolute shrinkage and selection operator (LASSO) (L1) penalty based on Cox (death) or } \\
\text { logistic (rash, haemoptysis, leukopenia) regression. The best-fitting model minimises prediction error in } \\
\text { leave-one-out cross-validation. In all models, } 49 \text { nonconstant virulence factors as well as the Charlson } \\
\text { comorbidity score were considered as potential predictors. Note that LASSO models do not define confidence } \\
\text { intervals. HR: hazard ratio; -: predictor not included in model. }\end{array}$} \\
\hline
\end{tabular}


inhibitor) was associated with both conditions, and the staphylococcal enterotoxin A gene (seA) gene predicted leukopenia.

\section{Discussion}

The present results improve our understanding of the role of PVL in different clinical presentation and severity of staphylococcal pneumonia. In addition, we identify the role of other severity-associated factors including bacteriological ones, and their link with PVL.

PVL-positive CAP and its severity were not evenly distributed according to age. PVL-negative CAP was virtually absent in toddlers and mortality was low. Many infectious processes vary in terms of presentation between young and older subjects, but the differences we observed exceed the expected variations due to age alone. For instance, radiological findings in pneumonia are usually similar at all ages [22]; however, we observed substantial radiological differences, notably regarding pleural effusion, pneumothorax and unilateral involvement that were all more frequent in toddlers. In addition, it was unexpected to observe a lower mortality in very young children compared to young adults without underlying conditions. The lower severity associated with PVL in toddlers could be due to 1) immunological immaturity, considering that local inflammation plays a central role in PVL-induced lesions [23]; 2) passive protection by maternal antibodies; or 3) the route of infection (the bullous lesions in toddlers point towards the involvement of the supporting tissue), suggesting a haematogenous route of infection as opposed to a probable inhalation route in adolescents and adults leading to direct necrosis of the respiratory epithelium. The observed specific symptoms and lower severity in toddlers might reflect a combination of one or more of the factors described, but also other features yet to be identified (see additional comments in the supplementary material). Thus, despite similarities in terms of site of infection and bacteriology, staphylococcal CAP in young children (i.e. pleuropneumonia) and staphylococcal necrotising pneumonia in adults should be considered as two distinct entities.

As we uncovered major differences according to age in PVL-positive staphylococcal pneumonia, the lack of consideration of age-specificity of the disease severity may at least partially explain some conflicting results reported in the literature $[14,24]$. Considering that PVL-negative staphylococcal pneumonia is almost absent in the youngest patients, toddlers should be excluded from analysis to assess the role of PVL in severity.

We observed that PVL was associated with specific symptoms in staphylococcal pneumonia of adolescents and adults. Previously described features strongly associated with PVL [2, 7] were observed herein among patients aged $\geqslant 3$ years: PVL-positive $S$. aureus pneumonia occurred mainly in younger people without underlying conditions and was associated with cutaneous rash, airway haemorrhage and leukopenia. However, logistic regression revealed that rash was most strongly predicted by the agrIV-CC121 genetic background, independently of PVL or the presence of superantigens. The basis of the association of PVL with rash appears elusive, although rash as a symptom remains a relevant marker of PVL-positive CAP.

Airway haemorrhage is a consequence of respiratory epithelial necrosis, as described in lung autopsies [1, 2]. Haemorrhage is an indirect effect of PVL, since the presence of polymorphonuclear (PMN) cells in the lung is required for PVL-induced necrotic lesions of the epithelium [25]. PMN cell influx to the lung may be generated by two nonexclusive mechanisms: a preceding viral infection producing chemoattractant for neutrophils by epithelial cells [25], and PVL-mediated inflammasome activation (releasing interleukin (IL)-1ß), leading to PMN cell recruitment and activation [26]. Interestingly, this inflammatory pathway is amplified by the release of pathogen- and damage-associated molecular patterns from dying neutrophils, possibly explaining the fulminant nature of PVL-associated CAP [27]. Of note, the PSM $\alpha 3-, \beta-, \gamma-$, $\delta$-haemolysins and LukDE have been shown to synergise with PVL to amplify IL-1 $\beta$ release, indicating that these factors cooperate with PVL to trigger inflammation [26]. However, among the factors listed above, LukDE is the only variable genotypic determinant and there was no direct association with haemoptysis in the LASSO model. Conversely, the LASSO model revealed that beside PVL, edinB was the second genotypic marker associated with haemoptysis, a feature not described previously. Edin-B elicits large transcellular tunnels in endothelial cells (macroapertures) inducing a loss of barrier function and providing direct access of the endothelium basement membrane to $S$. aureus. In mouse models, Edin-B promotes the translocation of $S$. aureus to the bloodstream during the course of pneumonia [28]. How these properties translate into haemoptysis remains to be explored.

We show that leukopenia is another major feature associated with PVL and death. The relationship between leukopenia and PVL has a mechanistic explanation since the identification of C5aR on myeloid cells as the receptor for lukS-PV [29]. Beside PVL, other determinants contributed to leukopenia, such as CC152 and CC398 lineage, Edin-B and SEA. This later association is novel in humans; it potentially 
results from extravasation of cells in a V $\beta$-unrestricted manner as demonstrated experimentally in rabbits for another major superantigen, the TSST-1 [30].

One objective of the present study was to assess the link between PVL and severity, considering that such an association has been controversial since the first description of PVL-associated pneumonia in 2002 [2]. The frequency of PVL in severe CAP in adults herein contrasts with the low frequency of PVL $(<5 \%)$ in the staphylococcal carriage population in France [17], and this striking difference constitutes the initial evidence for an association of PVL with severity. We observed that PVL, especially but not exclusively when associated with methicillin resistance, was an independent factor of mortality in staphylococcal CAP even when other virulence markers were considered, outweighing protective factors such as young age and the absence of underlying conditions. The present study indicates that PVL is associated with nonspecific severity markers of infection such as elevated lactate or presence of septic shock at admission and absence of reduction in lactate between admission and day 1 . All these factors are associated with a higher mortality in sepsis, irrespective of the bacteria involved [31, 32], and their higher frequency in the PVL-positive cases confirms greater severity in such patients. Surrogate markers of respiratory failure, such as the need for nitric oxide and for ECMO, were more frequent in PVL-positive cases, which also indicates greater severity in accordance with recent studies [33, 34]. The major symptoms associated with PVL (i.e. airway haemorrhage and leukopenia) were associated with mortality in the multivariable prediction model (table 4), strongly suggesting that these factors mediate the link between PVL and death.

Since its first description [2], PVL-positive S. aureus pneumonia has been reported worldwide [34-37]. A particular situation is observed in the United States where the description of necrotising pneumonia coincided with the emergence of the PVL-positive CA-MRSA USA300 lineage [4, 38]. PVL has been also associated with the CA-MRSA ST-80 lineage in Europe [39]. However, only 11 and five strains of the present study belong to these clones, respectively, and PVL was distributed within a large diversity of genetic backgrounds (i.e. $13 \mathrm{CCs}$ ) with a majority of methicillin-susceptible S. aureus, ruling out a possible clonal bias associated with PVL-positive cases. The lack of genetic diversity of CA-MRSA in the US may have initially led to confusion in the understanding of the determinant of severity associated with staphylococcal CAP. Thus, methicillin resistance was thought to be the prominent determinant for severity, presumably by inducing a delay in initiation of appropriate antibiotics, in line with our observations. However, this higher mortality in MRSA was present both in PVL-positive and PVL-negative pneumonia patients.

Mortality increased at ages $>60$ years (markedly in PVL-negative cases), which is anticipated for bacterial pneumonia; however, mortality reached a plateau in PVL-positive cases at $\sim 30$ years, an observation for which we have only hypothetical and nonexhaustive explanations. Mortality results from a balance of protective and deleterious factors. Protection is probably conferred by a gradual acquisition of toxin neutralising and opsonising antibodies against $S$. aureus. Conversely, enhanced severity may be caused by a highly reactive innate immune system (typically lacking in toddlers). Alternatively, the age-dependent acquisition of autoantibodies against cytokines and chemokines involved in S. aureus response (IL-1B, tumour necrosis factor- $\alpha$, macrophage inflammatory protein- $1 \alpha$, granulocyte-macrophage colony-stimulating factor, IL-17A and interferon (IFN)- $\gamma$ [40]) may impair protective immune responses in a subset of young adults, mimicking the immunosuppression associated with autoantibodies against type I IFNs in patients with severe severe acute respiratory syndrome coronavirus 2 [41].

We acknowledge limitations of the present study, most notably the observational design, whereby all participating centres were encouraged to enrol patients fulfilling the inclusion criteria. We cannot exclude under-reporting, and a higher reporting of the most severe cases may have occurred, but this is unlikely to have affected directly the comparison between PVL-positive and PVL-negative cases. The number of missing data were limited and exhaustivity was reached for all major parameters. Furthermore, the study was restricted to France, but covers the French territory almost entirely with 77 participating centres, thus limiting possible local epidemiological bias.

In conclusion, the present study demonstrates the association of PVL with two distinct facets of staphylococcal CAP with marked differences between toddlers and adolescents/adults regarding clinical presentation and outcome. In toddlers, PVL appears to be prominent in staphylococcal pneumonia, presentation matches with pleuropneumonia, and standard of care in modern ICU appears to be sufficient for favourable outcome. In contrast, PVL-positive CAP in adolescents and adults remains extremely severe despite aggressive management; it deserves further research to develop tailored therapeutic approaches. 
Acknowledgements: We thank the technicians of the French Reference Centre for Staphylococci for their contribution in characterising S. aureus isolates. We thank Vincent Cottin (Department of Respiratory Medicine, Louis Pradel Hospital, Hospices Civils de Lyon, Lyon, France ), Etienne Javouhey (Réanimation Pédiatrique, Hôpital Femme-Mère-Enfant, Hospices Civils de Lyon) and Thomas Henry (Centre International de Recherche en Infectiologie (CIRI), Lyon) for fruitful discussions. Finally, we thank Philip Robinson (DRCl, Hospices Civils de Lyon) for copyediting the manuscript.

The PVL pneumonia study group: Olivier Baldesi and Nathalie Brieu, Aix-En-Provence; Gérald Boussicault, Marie Kempf and Carole Lemarie, Angers; Valérie Charbonneau, Arnaud Desachy, Caroline Garandeau and François Pettinelli, Angouleme; Stéphane Bland and David Bougon, Annecy; Anne-Marie Colin-Gorski, Laurence Courdavault, Hervé Mentec and Agnès Scanvic, Argenteuil; Marie-Pierre Lacomme and Jean-Pierre Zanini, Aubertvilliers; Alexandre Avenel, Fabrice Bertrand, Elodie Colin and Huong Porcheret, Aulnay-Sous-Bois; Laurence Delapierre and Cécile Jensen, Avignon; David Leyssene and Jérôme Pillot, Bayonne; Émilie Benabid and Jack Richecoeur, Beaumont Sur Oise; Katy Jeannot, Anaïs Potron and Olivier Schulze, Besancon; Magali Anastay and Juliette Chabrol, Bethune; Christophe Clec'h and Mathilde Lescat, Bobigny Avicenne; Henri De Montclos and Xavier Tchenio, Bourg-En-Bresse; Frédéric Brun, Philippe Crova and Charlotte Tellini, Bourgoin-Jallieu; Bianca Podac and Martial Delorme, Chalon Sur Saone; Emmanuelle Lavalard and Salem Ould Zein, Chalons En Champagne; Marion Levast and Matteo Miquet, Chambery; Olivier Gontier, Abdelkader Ouchenir and Oana Zamfir, Chartres; Geneviève Barjon and Jean-Philippe Edmond, Compiegne; Guillaume Chevrel, Sabina Djouhri and Philippe Cormier, Corbeil-Essones; Said Aberrane and Rusel Leon, Creteil; Julien Bador, Pierre-Emmanuel Charles, Catherine Neuwirth and Jean-Pierre Quenot, Dijon; Caroll Barthalay-Le Risbe, Annick Bosseray, Barbara Colombe, Armelle Gayot, Christine Recule, Sébastien Thomas and Pascale Verger, Grenoble; Sylvaine Bastian and Pascale Piednoir, Guadeloupe Pointe A Pitre; Ahmed-Said Laggoune and Odile Sevin, Gueret; Henri Faure, Karine Grenet and Philippe Martinais, Jossigny; Laurent Bellec, Olivier Belmonte and Marie Lagrange-Xelot, La Reunion Saint-Denis; Emmanuel Antok, Julien Cousty and Sandrine Picot, La Reunion Saint-Pierre; Hélène Biessy and Alexandre Herbland, La Rochelle; Céline Ramanantsoa and Patrice Tirot, Le Mans; François Fourrier and Nadine Lemaitre, Lille; Elodie Couve Deacon, Deborah Postil and Philippe Vignon, Limoges; Pierre-Eric Danin, Oana Dumitrescu, Arnaud Friggeri and Elisabeth Hodille, Lyon CHLS; Bertrand Delannoy, Coralie Bouchiat, Céline Dupieux-Chabert, Frédéric Laurent, Sylvestre Tigaud and Grégoire Wallon, Lyon Croix Rousse; Etienne Javouhey, Yves Gillet and Anne Tristan, Lyon HFME; Patrick Gerome, Floriane Klack, Christophe Lions, Fabrice Petitjeans, Marc Puidupin and Marie-Pierre Otto, Lyon Hia Desgenettes; Laurent Argaud, Coralie Bouchiat, Martin Cour, Romain Hernu, Julien Illinger and François Vandenesch, Lyon Hôpital Edouard Herriot; Matthias JacquetLagreze and Laure Olivier-Soubirou, Lyon Louis Pradel; Arnaud Francois, Lionel Liron, Lyon Tonkin; Anne Carta and Marc Houvenaeghel, Marignane Clinique Générale; Grégory Dubourg, Nadim Cassir, Frédérique Gouriet, Philippe Minodier, Claire Nicaise and Laurent Papazian, Marseille APHM; Jean-Louis Ferge, Claude Olive and Rafaelle Theodose, Martinique Fort De France; Abdourahim Chamouine, Louis Collet, Philippe Durasnel and Thierry Benoit-Cattin, Mayotte; Françoise Crepet and Mélanie Crozet, Montbrison; Patrick Marthelet and Gilles Rival, Montelimar; Helene Jean Pierre and Camille Maury, Montpellier; Philippe Linval and Aina Raobison, Moulin Yzeure; Thierry Conroy, Nicolas Ducrocq, Serge Le Tacon, Pierre Perez and Cynthia Pianetti, Nancy; Michaël Afanetti, Nicolas Clement, Nicolas Degand, Jean Dellamonica and Sophie Leotard, Nice; Faten El Sayed, Xavier Repesse, Valérie Sivadon-Tardy, Nathalie Soismier and Valérie Soussan-Banini, Paris Hôpital Ambroise Paré; Christelle Guillet-Caruba and Pierre Trouiller, Paris Hôpital Antoine Béclère; Etienne De Montmollin and Etienne Ruppe, Paris Hôpital Bichat-Claude Bernard; Loic De Pontual and Isabelle Poilane, Paris Hôpital Jean-Verdier; Rishma Amarsy Guerle and Joaquim Mateo, Paris Hôpital Lariboisière; Mikael Alves and Jacques Tankovic, Paris Hôpital St Antoine; Jean-Luc Donay and David Schnell, Paris Hôpital St Louis; Mouna Doufair, Muriel Fartoukh, Antoine Parrot, Tai Pham, Charlotte Verdet and Sophie Vimont-Billarant, Paris Hôpital Tenon; Alexandra Aubry, Jean Chastre, Yannick Costa, Delphine Desbois, Sylvie Dubourdieu, Guillaume Hekimian, Antoine Landivier, Emilie Lafeuille, Ania Nieszkowska, Florence Richardin and Maxime Thouvenin, Paris Pitié Salpétrière; Stéphane Bonacorsi, Chantal Chaplain, Stéphane Dauger, Mathie Lorrot and Patricia Mariani-Kurkdjian, Paris Robert Debré; Chantal Chaplain, Vincent loos, Nathalie Memain and Bruno Verdiere, Paris Saint-Denis; Philippe Badia, Aude Bourrouillou, Gisèle Mourin and Cristel Voinot, Pau; Pierre-Guy Durand and Philippe Gueudet, Perpignan; Caroline Piau-Couapel, Samer Kayal, Pierre Tattevin and Fabrice Uhel, Rennes; Pascal Beuret and Michelle Boyer, Roanne; Quentin Blanc and Jean-Claude Colombani, Romans Sur Isere; Anne Vachee and Clément Vanbaelinghem, Roubaix; Kevin Guernon, Jean-Louis Pons, Christophe Girault and Ludovic Lemee, Rouen; Anne Carricajo, Florence Grattard, Richard Jospe, Pierre Lafond, Stephanie Rapin and Emmanuelle Rolland, Saint-Etienne; Anne-Marie Camin-Ravenne, Thierry Dulac and Alain Le Coustumier, Tarbes; Maryse Archambaud and Jean-Marie Conil, Toulouse Rangueil; Kildie Barrial and Patrice Fernandez, Valence; Julien Huntzinger and Nathalie Prades, Vannes; Fabrice Bruneel and Eric Farfour, Versailles, Hôpital Mignot; Jamal Bouhaddouch, Kevin Chaulier, Elodie Dorangeon and Camille Piffaut, Villefranche Sur Saone; Philippe Labadie and Bruno Soullie, Villenave D'ornon Hia Robert Picqué.

Author contributions: Concept and design: Y. Gillet, L. Argaud and F. Vandenesch; acquisition, analysis or interpretation of the data: Y. Gillet, A. Tristan, M. Saadatian-Elahi, C. Bouchiat, O. Dumitrescu, M. Leloire, 
C. Dupieux, F. Laurent, G. Lina, J. Etienne, P. Vanhems, L. Argaud and F. Vandenesch; statistical analysis: J-P. Rasigade and P. Vanhems; drafting of the manuscript: Y. Gillet, A. Tristan, J-P. Rasigade and F. Vandenesch; critical revision of the manuscript for important intellectual content: all authors; obtained funding: $F$. Vandenesch; administrative, technical or material support: M. Bès and C. Dupieux; study supervision: M. Saadatian-Elahi and A. Tristan.

Conflict of interest: Y. Gillet has nothing to disclose. A. Tristan has nothing to disclose. J-P. Rasigade reports personal fees from MSD and Pfizer, grants from bioMérieux, outside the submitted work. M. Saadatian-Elahi has nothing to disclose. C. Bouchiat has nothing to disclose. M. Bès has nothing to disclose. O. Dumitrescu has nothing to disclose. M. Leloire has nothing to disclose. C. Dupieux has nothing to disclose. F. Laurent has nothing to disclose. G. Lina has nothing to disclose. J. Etienne has nothing to disclose. P. Vanhems reports personal fees from Astellas, Biosciences and Pfizer, grants from Anios and MSD, outside the submitted work. L. Argaud has nothing to disclose. F. Vandenesch has nothing to disclose.

Support statement: The study was funded by French Ministry of Health under the Programme Hospitalier de Recherche Clinique 2011 (grant number 2010-A01132-37). This report presents independent research funded by the Ministry of Health; the views and opinions expressed in this publication are those of the authors and do not necessarily reflect those of the Ministry of Health. Funding information for this article has been deposited with the Crossref Funder Registry.

\section{References}

$1 \quad$ Chickering HT, Park JHJ. Staphylococcus aureus pneumonia. JAMA 1919; 72: 617-626.

2 Gillet $\mathrm{Y}$, Issartel B, Vanhems $\mathrm{P}$, et al. Association between Staphylococcus aureus strains carrying gene for Panton-Valentine leukocidin and highly lethal necrotising pneumonia in young immunocompetent patients. Lancet 2002; 359: 753-759.

3 Wollenman OJ, Finland M. Pathology of staphylococcal pneumonia complicating clinical influenza. Am J Pathol 1943; 19: 23-41.

4 Centers for Disease Control and Prevention (CDC). Four pediatric deaths from community-acquired methicillin-resistant Staphylococcus aureus - Minnesota and North Dakota, 1997-1999. MMWR Morb Mortal Wkly Rep 1999; 48: 707-710.

5 Lina G, Piémont Y, Godail-Gamot F, et al. Involvement of Panton-Valentine leukocidin-producing Staphylococcus aureus in primary skin infections and pneumonia. Clin Infect Dis 1999; 29: 1128-1132.

6 David MZ, Daum RS. Community-associated methicillin-resistant Staphylococcus aureus: epidemiology and clinical consequences of an emerging epidemic. Clin Microbiol Rev 2010; 23: 616-687.

7 Kreienbuehl L, Charbonney E, Eggimann P. Community-acquired necrotizing pneumonia due to methicillin-sensitive Staphylococcus aureus secreting Panton-Valentine leukocidin: a review of case reports. Ann Intensive Care 2011; 1: 52.

8 Li H-T, Zhang T-T, Huang J, et al. Factors associated with the outcome of life-threatening necrotizing pneumonia due to community-acquired Staphylococcus aureus in adult and adolescent patients. Respiration 2011; 81: 448-460.

9 Mandell LA, Wunderink R. Methicillin-resistant Staphylococcus aureus and community-acquired pneumonia: an evolving relationship. Clin Infect Dis 2012; 54: 1134-1136.

10 Hendren WH, Haggerty RJ. Staphylococcic pneumonia in infancy and childhood; analysis of seventy-five cases. J Am Med Assoc 1958; 168: 6-16.

11 Rebhan AW, Edwards HE. Staphylococcal pneumonia: a review of 329 cases. Can Med Assoc J 1960; 82: 513-517.

12 Carrillo-Marquez MA, Hulten KG, Hammerman W, et al. Staphylococcus aureus pneumonia in children in the era of community-acquired methicillin-resistance at Texas Children's Hospital. Pediatr Infect Dis J 2011; 30: 545-550.

13 Lemaître C, Angoulvant F, Gabor F, et al. Necrotizing pneumonia in children: report of 41 cases between 2006 and 2011 in a French tertiary care center. Pediatr Infect Dis J 2013; 32: 1146-1149.

14 Shallcross LJ, Fragaszy E, Johnson AM, et al. The role of the Panton-Valentine leucocidin toxin in staphylococcal disease: a systematic review and meta-analysis. Lancet Infect Dis 2013; 13: 43-54.

15 Bubeck Wardenburg J, Palazzolo-Ballance AM, Otto M, et al. Panton-Valentine leukocidin is not a virulence determinant in murine models of community-associated methicillin-resistant Staphylococcus aureus disease. $J$ Infect Dis 2008; 198: 1166-1170.

16 Diep BA, Gillet Y, Etienne J, et al. Panton-Valentine leucocidin and pneumonia. Lancet Infect Dis 2013; 13: 566.

17 Tristan A, Rasigade J-P, Ruizendaal E, et al. Rise of CC398 lineage of Staphylococcus aureus among infective endocarditis isolates revealed by two consecutive population-based studies in France. PLoS One 2012; 7 : e51172. 
18 van der Heijden GJMG, Donders ART, Stijnen T, et al. Imputation of missing values is superior to complete case analysis and the missing-indicator method in multivariable diagnostic research: a clinical example. $J$ Clin Epidemiol 2006; 59: 1102-1109.

19 Simon N, Friedman J, Hastie T, et al. Regularization paths for Cox's proportional hazards model via coordinate descent. J Stat Softw 2011; 39: 1-13.

20 Gillet $\mathrm{Y}$, Vanhems $\mathrm{P}$, Lina $\mathrm{G}$, et al. Factors predicting mortality in necrotizing community-acquired pneumonia caused by Staphylococcus aureus containing Panton-Valentine leukocidin. Clin Infect Dis 2007; 45: 315-321.

21 Richiardi L, Bellocco R, Zugna D. Mediation analysis in epidemiology: methods, interpretation and bias. Int $J$ Epidemiol 2013; 42: 1511-1519.

22 Patterson CM, Loebinger MR. Community acquired pneumonia: assessment and treatment. Clin Med 2012; 12: 283-286.

23 Diep BA, Chan L, Tattevin P, et al. Polymorphonuclear leukocytes mediate Staphylococcus aureus PantonValentine leukocidin-induced lung inflammation and injury. Proc Natl Acad Sci USA 2010; 107: 5587-5592.

24 Diep BA, Palazzolo-Ballance AM, Tattevin P, et al. Contribution of Panton-Valentine leukocidin in community-associated methicillin-resistant Staphylococcus aureus pathogenesis. PLoS One 2008; 3: e3198.

25 Niemann S, Ehrhardt C, Medina E, et al. Combined action of influenza virus and Staphylococcus aureus Panton-Valentine leukocidin provokes severe lung epithelium damage. J Infect Dis 2012; 206: 1138-1148.

26 Perret M, Badiou C, Lina G, et al. Cross-talk between Staphylococcus aureus leukocidins-intoxicated macrophages and lung epithelial cells triggers chemokine secretion in an inflammasome-dependent manner. Cell Microbiol 2012; 14: 1019-1036.

27 Holzinger D, Gieldon L, Mysore V, et al. Staphylococcus aureus Panton-Valentine leukocidin induces an inflammatory response in human phagocytes via the NLRP3 inflammasome. J Leukoc Biol 2012; 92: 1069-1081.

28 Courjon J, Munro P, Benito Y, et al. EDIN-B promotes the translocation of Staphylococcus aureus to the bloodstream in the course of pneumonia. Toxins 2015; 7: 4131-4142.

29 Spaan AN, Henry T, van Rooijen WJM, et al. The staphylococcal toxin Panton-Valentine leukocidin targets human C5a receptors. Cell Host Microbe 2013; 13: 584-594.

30 Waclavicek M, Stich N, Rappan I, et al. Analysis of the early response to TSST-1 reveals VB-unrestricted extravasation, compartmentalization of the response, and unresponsiveness but not anergy to TSST-1. J Leukoc Biol 2009; 85: 44-54.

31 Singer M, Deutschman CS, Seymour CW, et al. The Third International Consensus Definitions for Sepsis and Septic Shock (Sepsis-3). JAMA 2016; 315: 801-810.

32 Casserly B, Phillips GS, Schorr C, et al. Lactate measurements in sepsis-induced tissue hypoperfusion: results from the Surviving Sepsis Campaign database. Crit Care Med 2015; 43: 567-573.

33 Gijón M, Bellusci M, Petraitiene B, et al. Factors associated with severity in invasive community-acquired Staphylococcus aureus infections in children: a prospective European multicentre study. Clin Microbiol Infect 2016; 22: 643.

34 Jacquot A, Luyt C-E, Kimmoun A, et al. Epidemiology of post-influenza bacterial pneumonia due to PantonValentine leucocidin positive Staphylococcus aureus in intensive care units: a retrospective nationwide study. Intensive Care Med 2019; 45: 1312-1314.

35 Hsu L-Y, Koh T-H, Anantham D, et al. Panton-Valentine leukocidin-positive Staphylococcus aureus, Singapore. Emerging Infect Dis 2004; 10: 1509-1510.

36 Holmes A, Ganner M, McGuane S, et al. Staphylococcus aureus isolates carrying Panton-Valentine leucocidin genes in England and Wales: frequency, characterization, and association with clinical disease. J Clin Microbiol 2005; 43: 2384-2390.

37 Francis JS, Doherty MC, Lopatin U, et al. Severe community-onset pneumonia in healthy adults caused by methicillin-resistant Staphylococcus aureus carrying the Panton-Valentine leukocidin genes. Clin Infect Dis 2005; 40: 100-107.

38 Finelli L, Fiore A, Dhara R, et al. Influenza-associated pediatric mortality in the United States: increase of Staphylococcus aureus coinfection. Pediatrics 2008; 122: 805-811.

39 Dufour P, Gillet Y, Bes M, et al. Community-acquired methicillin-resistant Staphylococcus aureus infections in France: emergence of a single clone that produces Panton-Valentine leukocidin. Clin Infect Dis 2002; 35 819-824.

40 Armentrout El, Liu GY, Martins GA. T cell immunity and the quest for protective vaccines against Staphylococcus aureus infection. Microorganisms 2020; 8: 1936.

41 Bastard P, Rosen LB, Zhang Q, et al. Autoantibodies against type I IFNs in patients with life-threatening COVID-19. Science 2020; 370: eabd4585. 